Ein Tag Notfalldienst ...

Nach dem Aufruf durch Prof. Hans Stalder, Genf, im Namen der Kommission «Recherche et réalisation en médecine appliquée» (RRMA) der SAMW, die jeden Text mit Fr. 500.- vergütet, erschienen in der Schweizerischen Ärztezeitung Nr. 10 vom 6. März 2002, publizieren wir hier weitere Texte. Informationen erhalten Sie unter folgender E-Mail-Adresse: hans.stalder@hcuge.ch.

Un jour de garde...

A la suite de l'invitation par le Professeur Hans Stalder, Genève, au nom de la Commission Recherche et réalisation en médecine appliquée RRMA de l'ASSM, qui rétribue les textes par Fr. 500.-, parue dans le Bulletin des médecins suisses no 10 du 6 mars 2002 , nous publions d'autres textes. Des informations peuvent être obtenues en écrivant à l'adresse e-mail suivante: hans.stalder@hcuge.ch.

\title{
Ein Tag Notfalldienst
}

\author{
H. R. Gehring
}

Korrespondenz:

Dr. med. Hansruedi Gehring Seminarstrasse 11

CH-3006 Bern
«Che dolore, che dolor!» Welch ein Schmerz, höre ich die Sizilianerin aus Bern-West von ihrem Wohnblock in die klirrend kalte Winternacht hinaus rufen, als wären wir in einer Verdioper. Wenigstens weiss ich jetzt, dass ich an der richtigen Adresse angekommen bin. Das stellt sich in gewissen Quartieren mit ihren verwinkelten Innenhöfen und Strassenabschnitten, die nicht einmal auf dem neuesten Stadtplan eingezeichnet sind, gelegentlich schwieriger dar als das Stellen der richtigen Diagnose. Ich denke an meine Praxisvertretungen auf dem Land zurück, wo die Bauern wenigstens eine Stallaterne an die Weggabelung stellten. Diesmal ist das Finden der Patientin, wie gesagt, kein Problem. Merkwürdig: Es quäle sie vermutlich ein Gallenstein, erzählt die alleinstehende Frau und es spricht vieles für eine Kolik. Doch der Schmerz lässt schon nach, als ich die Vene punktiert, aber das Spasmolytikum noch gar nicht inijziert habe. Geht es ihr schon besser, weil sie sich nun auf die Erleichterung verlassen kann? Ein Plazeboeffekt? Mein Versuch, sie mit ein paar Worten über ihre Heimatstadt Caltanissetta aufzuheitern, geht daneben. Dort unten fühle sie sich noch weniger zu Hause als hier, sagt sie. Die hätten doch keine Kultur, keiner ihrer Verwandten läse je ein Buch. Erst jetzt entdecke ich auf ihrem Nachttisch einen Roman von Carmelo Samonà, dem sizilianischen Dichter.

«Ist die Literatur vielleicht ihre eigentliche Heimat?»

«a, Sie haben recht! Und es geht mir schon ein wenig besser.»

Diese Begegnung fand noch zu der Zeit statt, als wir Psychiater in Bern uns am allgemeinen Notfall- dienst beteiligten. Hier konnte ich die als Assistent am Bezirksspital, auf Passagierschiffen und als Leiter einer Drogenberatungsstelle gesammelten Erfahrungen in somatischer Medizin anwenden. Noch einmal war ich ein «richtiger Doktor». Doch $80 \%$ der Notfallbesuche, die ich in der Bundesstadt ausführte, betrafen psychosomatische Situationen.

Während ich auf dem Heimweg von der Sizilianerin zu meiner Wohnung im Osten der Stadt auf Glatteis achtgeben muss, bekomme ich bei der Autobahnausfahrt von der Schwester des Ärztenotrufs den nächsten Auftrag: Die 78jährige Frau L. im Kirchenfeld leide an einem akuten Hustenanfall. Ihr Hausarzt sei schon in den Weihnachtsferien, ihre Tropfen wären ihr ausgegangen und sie könne unmöglich warten, bis um acht die Quartierapotheke öffne. Ein Fall für den Notfallarzt? Im letzten Dienst wurde ich um diese Zeit zu einem Fixer gerufen, dem nur das Geld fehlte, um das Taxi in sein Aussenquartier zu bezahlen. Das gehört vielleicht auch zur bio-psycho-sozialen Medizin. Inzwischen hat ein Nieselregen eingesetzt, Gefahr von Eisglätte. Frau L. wohnt im zweiten Stockwerk eines Mietshauses. Da ich ein bisschen farbenblind bin, kann ich nachts nicht unterscheiden, ob es rosarot oder sienabraun gestrichen ist. Sie schmettert keine Arie aus dem Fenster, dafür geben ihre zwei Yorkshireterrier an. Im Treppenhaus der muffige Geruch dieser Vorkriegshäuser. Die Wohnungstüre steht schon einen Spalt breit offen. Dahinter begrüssen mich Rico und Wady, die schnuckeligen Hunde. Frau L. liegt auf einem Biedermeiersofa und hüstelt. Ob sie nicht aufsitzen wolle? Sie sieht mich bedeutungsvoll an und zeigt zur abblätternden Decke. Da ich niessen muss, die 
feuchte Kälte dieser Nacht setzte mir schon ein bisschen zu, bietet sie mir einen Kräuterschnaps an, der meine Eingeweide zusammenzieht.

In der offenen Schublade eines ovalen Kommödchens hortet sie ihre Medikamente. Wenn ich schon Arzt sei, könne ich die wenigstens für sie ordnen. Ich stelle fest, dass die Hustentropfen nicht nur verfallen, sondern wegen ihres Kodeingehaltes längst nicht mehr im Handel sind. Sie fordert mich auf, ihr etwas anderes zu verschreiben. Ich gebe ihr aus meinem Koffer ein Fläschchen Resyltropfen. Sie bedankt sich und macht mich erneut auf den Mieter im oberen Stockwerk aufmerksam. Ihre Augen glühen noch inniger und ihr Gesicht drückt Verachtung aus:

«Das ist eine 〈Plackerei», kann ich Ihnen sagen, dauernd versucht er, mir seine Gedanken aufzuzwingen!»

«Was für Gedanken?» frage ich so arglos wie möglich.

«Die sind viel zu ekelhaft, um mit Ihnen darüber zu reden. Sie würden sich grün und blau ärgern.»

Leicht gebückt auf dem Sofa sitzend, hält sie sich an der Seitenlehne fest. Wenn sie sich ereifert, wirkt sie mächtiger als nach ihrer Statur: «Ich lasse mich von einem Santschi nicht fertigmachen, diese 〈Gewissensbelastung〉 ist nicht zum Aushalten», sagt sie und krault zwischendurch ihre Hunde, die während des Gesprächs mehrmals gegen die Zimmerdecke kläffen und panikartig fliehen, als man von oben tatsächlich etwas hört. Frau L. hustet immer noch, ich sehe rundherum gefüllte Aschenbecher. Sie führt mich in die Küche, wo sich Rico und Wady in den aus Draht und einer Asbestplatte gebastelten «Schutzkäfig» verkrochen haben, aus dem sie mir winselnd ihre Nasen entgegenstrecken.

«Gibt es einen gemeinsamen Wahn zwischen Mensch und Tier?» frage ich mich, während ich die alte Dame auf ihrem Biedermeiersofa perkutiere und auskultiere.

«Ich weiss, ich rauche zuviel, geradezu manisch!» sagt sie, «aber dieser Santschi zwingt mich dazu.»

«Ich hoffe, dass Ihre Hündchen nicht zuviel leiden», wage ich beim Abschied zu sagen.

«Gelt, die werden auch von Stimmen geplagt!»

$\mathrm{Da}$ die Halbwertszeit unseres medizinischen Wissens bekanntlich sehr kurz ist, war ich mit den Jahren doch froh, wenn ich mich mit den internistischen Kollegen, die sich mit mir den 48stündigen
Notfalldienst teilten - der eine auf der linken, der andere auf der rechten Seite der Aare -, dahingehend absprechen konnte, dass sie die 20\% der wirklichen Notfälle und ich dafür den bio-psycho-sozialen Part übernahm, unabhängig davon, an welchem Aareufer die Hilfe gefragt war. Natürlich konnte die Grenzlinie am Telefon nicht immer haarscharf gezogen werden. Darum profitierte ich von den militärmedizinischen Kursen, die eine minimale Fortbildung in Notarztmedizin anboten. Die Unikliniken hatten damals für unser Anliegen noch kein Gehör.

Diesmal ist mein Kollege ein Gynäkologe, der von zwei älteren Krankenschwestern seines Belegspitals um Hilfe gebeten wird. Bei ihnen wohne in der Mansarde ein Chemiestudent. Heute früh habe dieser Mann den Spiegel über dem Lavabo lautstark in kleine Stücke zerschlagen, berichtete mir Schwester Benedikte, und sei nun völlig verwirrt. Obwohl mir auf der Heimfahrt von Frau L. schon der feine Brötchenduft der Bäckerei Reinhardt in die Nase stach und ich mich auf ein Frühstück mit meiner Frau mit Kaffee und frischen Gipfeli freute, fahre ich zu den Personalhäusern dieses Spitals. Hatte nicht Friedrich Dürrenmatt als Student in einer dieser Mansarden gewohnt? Auf das Durcheinander in seiner Bude zeigend, begrüsst mich der Patient mit dem vielsagenden Satz: «Hier sehen Sie meine Identität!» Sein Gesicht ist mit Asche verschmiert und seinen Körper hat er wie ein indischer Sadhu in ein oranges Tuch gehüllt. In einer feierlichen Zeremonie überreicht er mir ein Glühlämpchen: «Und Sie sind meine Erleuchtung!» Erst jetzt bemerke ich die in hundert Rädchen und Federn zerlegte Uhr auf dem Tisch und die zu einem Kreuz angeordneten Bücher am Boden. Die Ständerlampe steht quer im Raum. Es herrscht ein unbeschreibliches Durcheinander, doch alles scheint einem höheren Sinn $\mathrm{zu}$ gehorchen. Zuoberst auf dem Bücherstapel liegt Manfred Bleulers Lehrbuch der Psychiatrie, aufgeschlagen beim Kapitel über Schizophrenie.

Die Tatsache, dass der Mensch an einer Psychose erkranken kann, war das Motiv, warum ich Psychiater wurde. Es geht hier um die Condition humaine. Darum macht für mich auch der Notfalldienst Sinn. Auf einer meiner Reisen als Gehilfe des Schiffsarztes lernte ich einen baltischstämmigen Allgemeinpraktiker aus New York kennen. Als ich bei ihm an einem vorgerückten Abend über unsere Notfalldienstpflicht jammerte, gab er mir kurz zurück: «My dear, that's our job!» 